\title{
Allergic bronchopulmonary disease caused by Curvularia lunata and Drechslera hawaiiensis
}

\author{
ROSE McALEER, DOROTHEA B KROENERT, JANET L ELDER, AND J H FROUDIST \\ From Medical Mycology Division, State Health Laboratories, and Department of Respiratory Medicine, \\ Sir Charles Gairdner Hospital, Perth, Western Australia
}

ABSTRACT Three patients who developed bronchoceles caused by fungi other than Aspergillus $s p$ are described. The first patient presented for investigation of a lesion at the right hilum on chest radiograph and a raised blood eosinophil count. A bronchogram showed complete block of the apical segmental bronchus which at operation was shown to be caused by inspissated material. The second patient was investigated because of a cough productive of plugs of sputum and irregular opacities in both upper zones on chest radiograph and a raised blood eosinophil count. This only cleared after one month on high dose oral prednisone therapy. The third patient with a previous history of left lingular pneumonia and bronchiectasis of the lingular segment of the left upper lobe was investigated three years later for right basal shadowing and a raised blood eosinophil count. The radiograph cleared after one month on high dose oral prednisone treatment. The aetiological agents in these cases were dematiaceous hyphomycetes, fungi ubiquitous in nature, and also agents of plant disease. The causal fungi, Curvularia lunata and Drechslera hawaiiensis, have on a few occasions been reported as causing human disease but in cases quite dissimilar to the three reported here. Septate branching dematiaceous mycelium was consistently seen in the clinical material and isolated from successive sputum specimens from each patient. Immunodiffusion tests from the third patient gave positive results for both fungi. Intraperitoneal inoculations of $C$ lunata and $D$ hawaiiensis into Swiss white mice proved the pathogenicity of these isolates.

Allergic bronchopulmonary aspergillosis as one cause of pulmonary eosinophilia is now a wellrecognised entity and the possibility that Candida may cause a similar syndrome has been suggested. ${ }^{1}$ Other fungi have seldom been implicated, although Dolan et $^{2}{ }^{2}$ reported two cases of bronchopulmonary disease caused by Helminthosporium species in two patients with asthma. The clinical course was similar to that of allergic aspergillosis. The three cases reported here are of bronchopulmonary disease caused by dematiaceous fungi closely related to Helminthosporium species, Curvularia lunata and Drechslera hawaiiensis ( $D$ hawaiiensis was previously classified as Helminthosporium hawaiiensis). Many species of the family dematiaceae are ubiquitous in nature. Because they are common in our environment they are found as contaminants in laboratory specimens but on some occasions have been agents of human and animal disease.

Address for reprint requests: Rose McAleer, Medical Mycology, State Health Laboratories, Box F312, GPO, Perth, Western Australia 6001.
$C$ lunata has been reported to be the aetiological agent in a case of mycetoma ${ }^{3}$ and also in a case of keratitis. ${ }^{4}$ Various other species of Curvularia have been reported as causing human disease. ${ }^{-7-7}$

$D$ hawaiiensis has been reported as the cause of human disease on two previous occasions, one a fatal case of meningo-encephalitis ${ }^{8}$ and the other of nasal obstruction. ${ }^{9}$ D spicifera was implicated in disease in a boy with acute lymphoblastic leukaemia, ${ }^{10}$ and there have been reports of $D$ rostata causing subcutaneous lesions in animals in Australia. ${ }^{11}{ }^{12}$ To our knowledge there have been no reports of bronchopulmonary disease in which either Curvularia or Drechslera species have been implicated.

\section{Mycological methods}

Sputa or sputum plugs from patients with suspected mycoses were examined by the mycology laboratory. When possible early morning specimens taken after rinsing the mouth with water were obtained and 
kept cool during transit to the laboratory. Four different microscopic preparations were examined from each specimen and cultures made for incubation at $26^{\circ} \mathrm{C}$ and $37^{\circ} \mathrm{C}$ on five different media including malt extract agar, brain heart infusion blood agar, and Sabouraud's dextrose agar.

Serology for Curvularia and Drechslera was not done for the first two patients because no established serological procedures existed for these infections at that time. However reagents were available to perform immunodiffusion tests for the third patient.

\section{Clinical reports}

\section{CASE 1}

A 33-year-old woman presented in January 1972 for investigation of a lesion at the right hilum detected in November 1971 at a routine mass radiography screening examination. She had a history of pneumonia with measles at the age of 12 years and subsequently occasional bronchitis. She was free of symptoms from the age of 16 years to 31 years when after the birth of her first child in 1969 she started to wake at night wheezing and a few weeks later developed a cough for which she was given antibiotic therapy. She had a further respiratory illness the following year and then remained well until after the birth of her second child in May 1971, when she developed a wheeze, dyspnoea, and minimal cough, which improved slightly with antibiotics. She frequently had a post-nasal discharge and on several occasions coughed definite dark yellow to brown plugs of sputum. She had occasional courses of antibiotics, and over the three months before presentation was well although she still produced occasional plugs of sputum after self-administered physiotherapy.

On admission to hospital in February 1972 she had coarse inspiratory and fine expiratory wheezes over the right anterior chest. A chest radiograph showed several discrete solid opacities at the right hilum (fig 1). Her vital capacity was 3.68 litres (predicted 3.36 l) and forced expiratory volume in one second 2.34 litres (predicted 2.80 1). After bronchodilator the recordings were vital capacity 3.78 litres and $\mathrm{FEV}_{1} 2.75$ litres. Her direct eosinophil count was 1080/cubic millimetre, and she had a sputum eosinophilia of $70 \%$. Prick skin tests gave a $3 \mathrm{~mm}$ reaction to Aspergillus fumigatus with a negative control; the precipitin test to Aspergillus was negative. She had negative skin tests to 19 other common allergens. Total IgE was not estimated. Bronchoscopy was performed and a small amount of mucoid sputum was seen in the orifice of the apical segment of the right lower lobe. A bronchogram showed complete block of the apical segmental bronchus of the right lower lobe (fig 2).

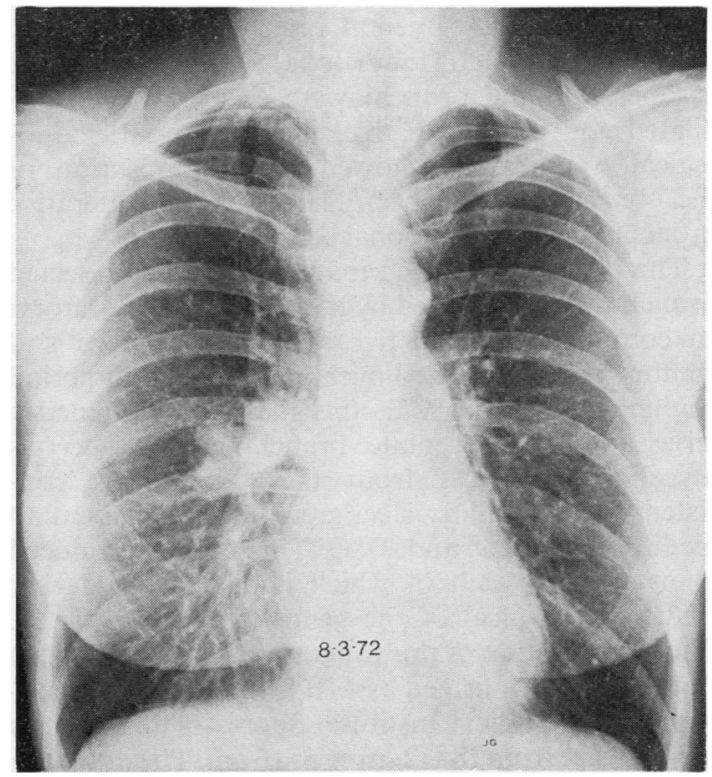

Fig 1 Case 1. Chest radiograph shows discrete solid opacities at the right hilum.

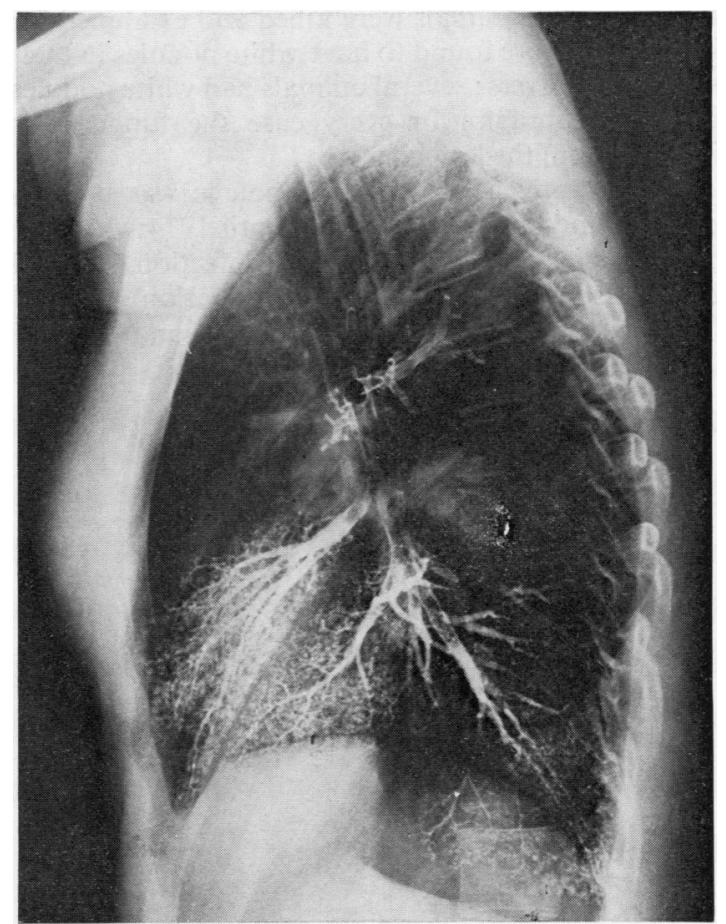

Fig 2 Case 1. Right bronchogram shows complete block of the apical segmental bronchus of right lower lobe. 
In April 1972 segmental resection of the apical segment of the right lower lobe was performed and at operation the bronchi were seen to be grossly dilated and filled with thick dark material. Pathological examination showed mucus impaction of the bronchi with necrotising and obliterative bronchiolitis and pulmonary granulomata.

The brown inspissated material from the resected bronchus was examined by the mycology laboratory. In common with the five sputum specimens and sputum plugs examined over the four-month period beginning January 1972, this material contained a large amount of septate branching dematiaceous mycelium. Cultures from these specimens consistently grew a fluffy steel green-grey fungus on all media at both $26^{\circ}$ and $37^{\circ} \mathrm{C}$. This fungus produced many conidia each of which had a larger darker central cell typical of the genus Curvularia (fig 3). The identification of this fungus as $C$ lunata (Walker) Boedijn, was confirmed by MB Ellis of the Commonwealth Mycological Institute. Spores of this species range in size from $18-32 \mu \mathrm{m} \times 8-16 \mu \mathrm{m}$. The optimum temperature of this isolate was $30^{\circ} \mathrm{C}$ and the maximum $40^{\circ} \mathrm{C}$.

To determine its pathogenicity this fungus was inoculated intraperitoneally into four Swiss white mice, following the technique of Fuste et al. ${ }^{8}$ After two weeks the animals were killed and examined for lesions. All were found to have white nodules in their spleens and livers; several animals had white nodules in the diaphragm. In every case the fungus was isolated from the lesions.

Postoperatively the patient's course was uncomplicated and she remained well until 1974 when she presented again with a respiratory infection followed by persistent dyspnoea. On examination she had inspiratory and expiratory wheezes and a vital capacity of 1.73 litres and $\mathrm{FEV}_{1}$ of 0.791 . There was no blood eosinophilia. Disodium cromoglycate and salbutamol therapy was instituted with symptomatic improvement within 48 hours. Her vital capacity had then risen to 3.07 litres and $\mathrm{FEV}_{1}$ to 2.12 litres thus confirming the diagnosis of asthma.

\section{CASE 2}

A 36-year-old woman presented in October 1975 with an upper respiratory tract infection followed by shortness of breath and a productive cough. She had a past history of allergic rhinitis since early childhood, exacerbated by housedust and flowers but no definite history of asthma. She lived in Broome in the northwest of Australia and in February 1975 an airconditioner was installed in her home. In May 1975 she developed a dry unproductive cough with the occasional expectoration of "pea-sized" yellow "cheese-like" plugs of sputum. Chest radiograph showed an irregular opacity in the right upper zone with a further opacity in the left upper zone. Appearances suggested mucoid impaction (fig 4). Blood eosinophil count was 4568 /cubic $\mathrm{mm}$ with sputum eosinophilia of $34 \%$. Prick tests were positive to $A$ fumigatus, Cladosporium, Alternaria, Helminthosporium, and grasses but negative to other allergens. Total IgE was $650 \mathrm{U} / \mathrm{ml}$ (normal less than $170 \mathrm{U} / \mathrm{ml}$ ). Precipitins and specific $\operatorname{IgE}$ against Aspergillus were negative. Her tests of respiratory function were vital capacity $3 \cdot 1$ litres (predicted 3.2 l), FEV $_{1}$ 2.70 litres (predicted 2.681 ). After bronchodilator, the recordings were vital capacity 3.0 litres and FEV $2 \cdot 65$ litres.

During the six weeks from 16 October to 21 November 1975 six sputa, some of which contained brown plugs, were examined and found to contain varying amounts of septate branching dematiaceous

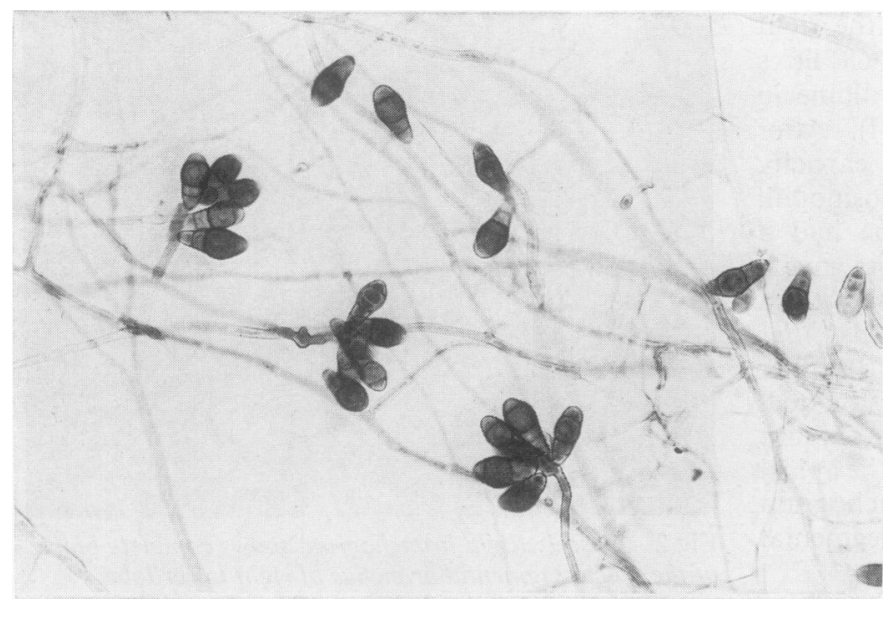

Fig 3 Conidophore and conidia of Curvularia lunata in a slide culture, original magnification $\times 850$. 


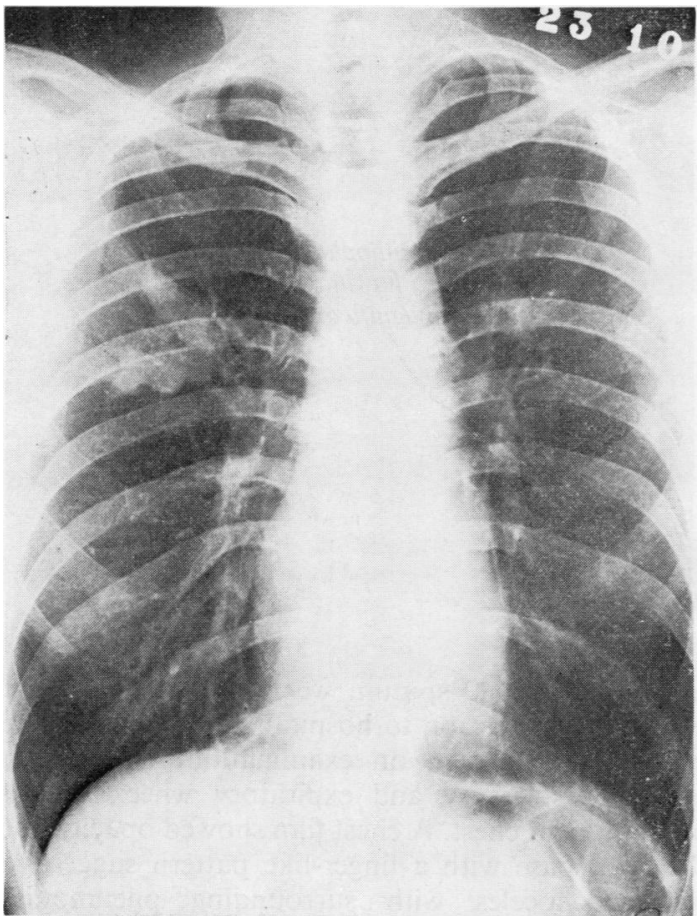

Fig 4 Case 2. Chest radiograph shows irregular opacity in the right upper zone with a further opacity in the left upper zone.

mycelium (fig 5). Culture of these specimens grew a fluffy grey fungus, at both $26^{\circ} \mathrm{C}$ and $37^{\circ} \mathrm{C}$ on all media. This fungus was originally thought to be Helminthosporium species because of its cylindrical multiseptate dematiaceous spores (fig 6) measuring 12-37 $\mu \mathrm{m} \times 5-11 \mu \mathrm{m}$. It was later confirmed by MB Ellis to be Drechslera hawaiiensis (Burgincourt, 1955) Subramanian and Jain ex Ellis 1966. This isolate had an optimum temperature of $26^{\circ} \mathrm{C}-30^{\circ} \mathrm{C}$ but grew well on all media up to $37^{\circ} \mathrm{C}$.

This fungus was similarly tested for pathogenicity to Swiss white mice and all four animals inoculated developed white nodules in their spleens and livers; two also had nodules in the diaphragm and one had adhesions on the spleen. The fungus was isolated from all lesions.

Dust samples taken from the filter of the air conditioner which had been installed in the patient's home five months earlier were examined and found to contain a large variety of fungal spores, including Drechslera type spores similar to those shown in fig 6.

She was treated with antibiotics and physiotherapy with improvement of symptoms but cough continued. Two bronchoscopies were performed. However, attempts to move past or aspirate the plugs were unsuccessful. Mucomyst was instilled and some bleeding occurred causing atelectasis of the subsegment of the right upper lobe with subsequent increased shadowing in the area. A bronchogram showed good filling of the entire bronchial tree; nevertheless despite intensive physiotherapy, regular nebulisations, and potassium iodide, no radiological improvement occurred and prednisone in a dose of $60 \mathrm{mg}$ daily was then given for two weeks and decreased to $40 \mathrm{mg}$ daily at the time of discharge. For two weeks after discharge she coughed up tiny brown plugs and chest radiograph after one month on the

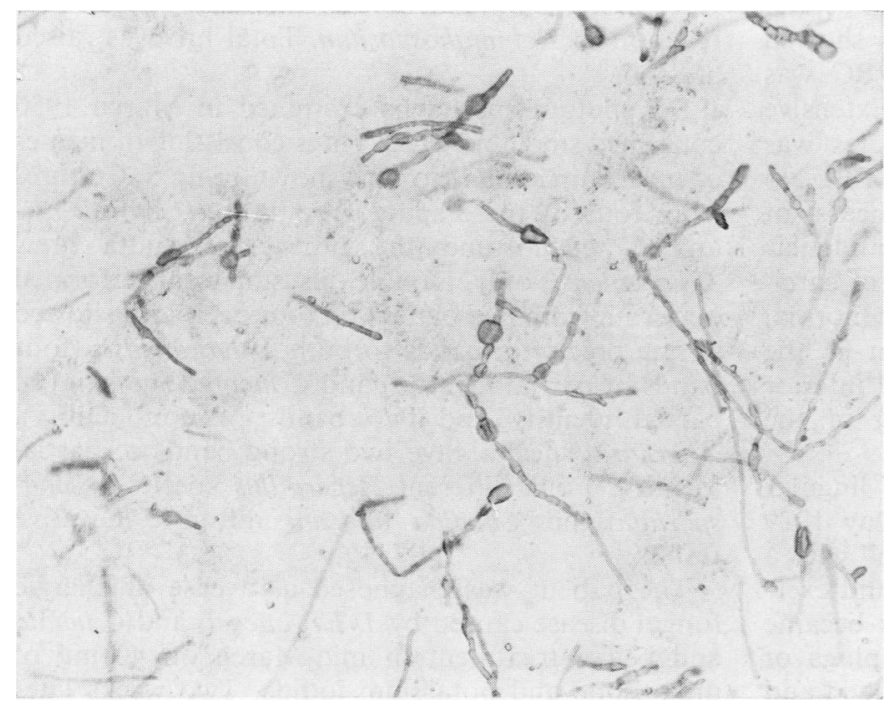

Fig 5 Dematiaceous mycelium of Drechslera hawaiiensis in sputum, original magnification $\times 850$. 


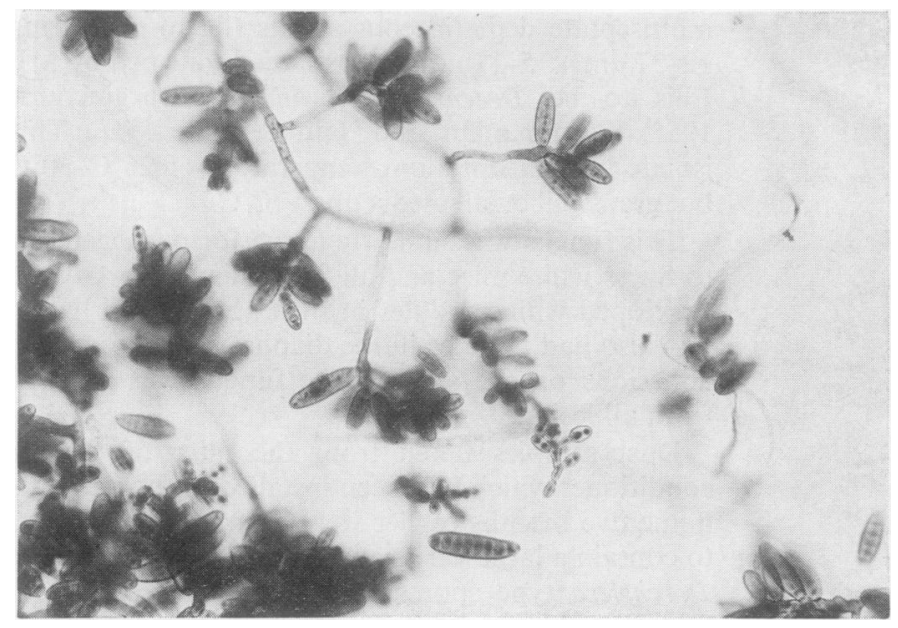

Fig 6 Conidiophores and conidia of Drechslera hawaiiensis in a slide culture, original magnification $\times 850$.

prednisone was clear. The prednisone was gradually decreased and she continued on a maintenance dose of $5 \mathrm{mg}$ daily.

Two years later in July 1977, two more sputum plugs from this patient were examined. They were sent from her home town of Broome, 2231 kilometres north of Perth. One contained masses of dematiaceous mycelium. When last seen in July 1979 she was well but still on a maintenance dose of prednisone.

\section{CASE 3}

A 35-year-old man, a non-smoker, first presented in September 1977 with sudden onset of haemoptysis and left shoulder pain. A chest radiograph showed lingular pneumonia. He improved on treatment with co-trimoxazole but in November 1977 he again had a brisk haemoptysis and was admitted to hospital for investigation. A chest radiograph showed consolidation in the lingular segment, WBC was 4100 with a normal differential, ESR $2 \mathrm{~mm}$. Extensive tests of lung function were normal. Mantoux test was negative and sputum was negative for AFB. No eosinophils were seen in cytological smears of sputum. Bronchoscopy showed a clot in the lingular bronchus and biopsy revealed no evidence of carcinoma. A bilateral bronchogram showed a normal right bronchial tree but proximal dilation of the second order bronchi of both superior and inferior lingular segments; there was poor filling of the bronchi in the periphery of these segments.

$\mathrm{He}$ was discharged with a diagnosis of lingular bronchiectasis and remained well until May 1979 when he again developed a "chest infection" with a cough productive of frothy white sputum and exertional dyspnoea. In October 1979 sputum became purulent and he also coughed grey firm plugs of sputum the size of grape seeds. This continued and in February 1980 sputum was flecked with bright blood. On admission to hospital in March 1980 the only abnormal sign on examination was a high pitched inspiratory and expiratory wheeze heard over the right chest. A chest film showed opacities at the right base with a finger-like pattern suggestive of bronchoceles with surrounding pneumonia. Blood eosinophil count was $1007 \mathrm{~mm}^{3}$, ESR $5 \mathrm{~mm}$. A plug of sputum contained moderate to large numbers of eosinophils. Vital capacity on admission was 5.07 litres (predicted 5.6 l) FEV $_{1} 4.08$ litres (predicted 4.41 ). Vital capacity on discharge was 5.43 litres and FEV 13.61 litres. No tests were done before and after bronchodilator on this admission. Prick skin tests were negative to 20 common allergens but showed a $3 \mathrm{~mm}$ reaction to $A$ fumigatus and $A$ terreus, a $4 \mathrm{~mm}$ reaction to Alternaria and a $5 \mathrm{~mm}$ reaction to Helminthosporium. Total IgE was raised at 8400 .

Six sputum specimens examined in March 1980 contained small brownish plugs consisting of masses of mycelium similar to that shown in fig 5. Cultures from four of these sputa grew both $D$ hawaiiensis and $C$ lunata and the other two sputa grew $D$ hawaiiensis only. Double diffusion tests performed on serum from this patient on four occasions showed strong precipitin bands to both $D$ hawaiiensis (four bands of partial identity) and $C$ lunata (one band of partial identity and two bands of non-identity). Alternaria species gave two strong bands of partial identity. Four different Aspergillus species, Cladosporium and Candida albicans all gave negative results.

The patient was diagnosed as a case of allergic fungal disease caused by $D$ hawaiiensis and $C$ lunata and began treatment in mid-March on $40 \mathrm{mg}$ of prednisone and potassium iodide. Two weeks later 
prednisone was increased to $60 \mathrm{mg}$ and this dose was continued for a further two weeks. A chest radiograph then showed marked clearing and prednisone was gradually reduced. When last seen he was well and the radiograph was normal except for cyst-like spaces in the right lower zone.

\section{Discussion}

The patients described in this report all had raised direct eosinophil counts with sputum eosinophilia and developed bronchoceles caused by fungi other than Aspergillus. They were not clinically recognised as having asthma at the time of diagnosis although the first patient had cough and wheeze for a short period in 1969 and developed clinical asthma two years after the diagnosis of fungal disease. Transient lung shadows were not demonstrated in the first two patients but both had bronchoceles at the time of diagnosis. The third patient also had no history of asthma and only one previous recognised episode of consolidation of the lingula yet a bronchogram at that time showed proximal bronchiectasis of the second order bronchi of both the superior and inferior lingular segments.

Both D hawaiiensis and C lunata are closely related dematiaceous hyphomycetes, fungi ubiquitous in nature and not uncommonly isolated from clinical specimens and air samples in this State. Most isolates have no significance, and it is only when mycelium is seen in the microscopy of clinical material and the same fungus isolated repeatedly, that any significance is given to their isolation. These fungi are readily inhaled from the atmosphere and this was the most likely source of the infection in our patients.
In Western Australia Curvularia species are common on senescing grasses and $C$ lunata has been recorded as a pathogen on sorghum at Kununurra (fig 7). Our first patient affected by this fungus developed her chest symptoms when living at Katherine in the Northern Territory. Curvularia species are ubiquitous in this area. D hawaiiensis has been isolated from birdwood grass grown at Derby, a town in the same semi-tropical part of the State as Broome, and it is probable that this fungus is present throughout the area. The third patient contracted his disease while working for three and a half years at Dampier, also in the northern part of the State. From this evidence it can be seen that the patients were living in areas where these fungi could have been present in the atmosphere.

Mullins and Seaton ${ }^{13}$ found that the presence of spores in the respiratory tract was related to their occurrence in the air. The spores of $C$ lunata and $D$ hawaiiensis are larger than those of fungi more commonly associated with lung disease. Spores of $A$ fumigatus are globose and measure up to $3.5 \mu \mathrm{m}$ in diameter, A niger $5 \mu \mathrm{m}$, and A flavus $6 \mu \mathrm{m}$. C lunata, and $D$ hawaiiensis have spores measuring from $18-32 \times 8-16 \mu \mathrm{m}$ and $12-37 \times 5-11 \mu \mathrm{m}$ respectively, the diameter usually being approximately one-third their length. Although these spores are large by comparison, still bigger spores have been isolated from lung specimens - that is, Stemphylium botryosum 27-42 $\times$ 24-30 $\mu \mathrm{m} .{ }^{13}$ Why these two species, of all the possible infective agents, acted as pathogens in these patients is not known.

We would like to thank Dr A Tribe for allowing us to present the third case.

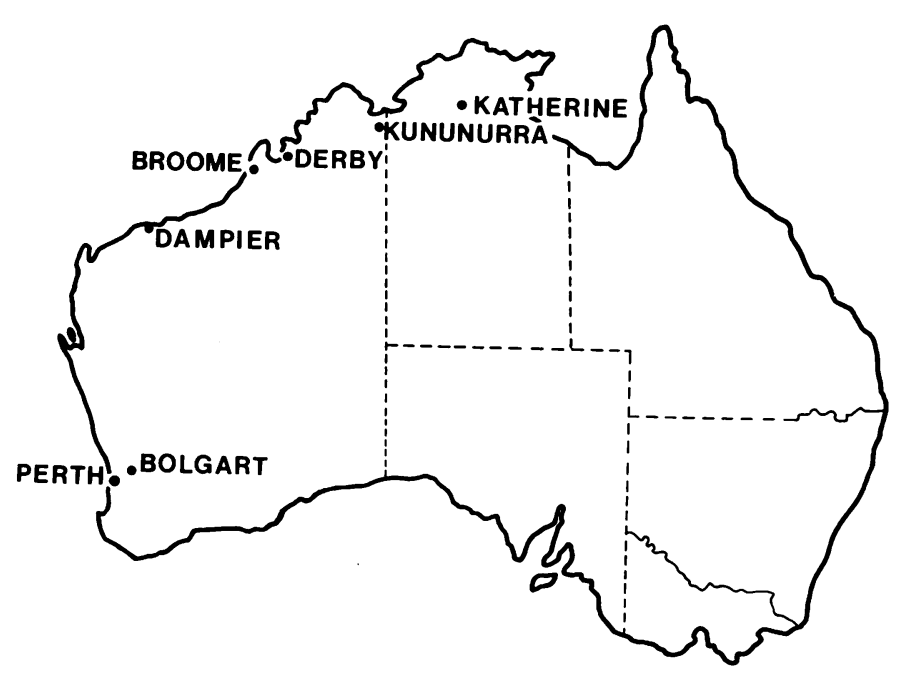

Fig 7 Map of Australia showing towns where patients live and also local areas where Curvularia lunata and Drechslera hawaiiensis have been isolated. 


\section{References}

1 Pepys J, Faux JA, Longbottom JL, McCarthy DS, Hargreave FE. Candida albicans precipitins in respiratory disease in man. $J$ Allergy $1968 ; 41: 305-18$.

2 Dolan CT, Weed LA, Dines DE. Bronchopulmonary helminthosporiosis. Am J Clin Pathol 1970; 53: $235-42$

3 Mahgoub ES. Mycetomas caused by Curvularia lunata, Madurella grisea, Aspergillus nidulans and Nocardia brasiliensis in Sudan. Sabourautia 1973; 11:179-82.

4 Nityananda K, Sivasubramaniam P, Ajello L. Mycotic keratitis caused by Curvularia lunata. Case report. Sabouraudia 1962; 2:35-9.

5 Kaufman SM. Curvularia endocarditis following cardiac surgery. Am J Clin Pathol 1971 ; 56:466-70.

6 Nityananda K, Sivasubramaniam P, Ajello L. A case of mycotic keratitis caused by Curvularia geniculata. Arch Opthalmol 1964; 71:456-8.
7 Lampert RP, Hutto JH, Donnelly WH, Shulman ST. Pulmonary and cerebral mycetoma caused by Curvularia pallescens. J Pediatr 1977; 91:603-5.

8 Fuste FJ, Ajello L, Threlkeld R, Henry JE. Drechslera hawaiiensis: causative agent of a fatal fungal meningo-encephalitis. 1973 Sabouraudia 11:59-63.

9 Young CN, Swart JG, Ackermann D, Davidge-Pitts K. Nasal obstruction and bone erosion caused by Drechslera hawaiiensis. J Laryngol Otol 1978; 92:137-43.

10 Estes SA, Merz WG, Maxwell LG. Primary cutaneous phaeohyphomycosis caused by Drechslera spicifera. Arch Dermatol 1977; 113:813-5.

11 McKenzie RA, Connole MD. Mycotic nasal granuloma in cattle. Aust Vet $J 1977 ; 53: 268-70$.

12 Pritchard D, Chick BF, Connole MD. Eumycotic mycetoma due to Drechslera rostata infection in a cow. Aust Vet J 1977; 53:241-4.

13 Mullins J, Seaton A. Fungal spores in lung and sputum. Clin Allergy 1978; 8: 525-33. 